\title{
Talent Identification and Development of Football Sport based on Player Position of PSGC 2018
}

\author{
Vila Elin Rusliani*, Dikdik Zafar Sidik, Dian Budiana \\ Universitas Pendidikan Indonesia \\ Bandung, Indonesia \\ *vilaelin26@gmail.com
}

\begin{abstract}
The main problem for any clubs is to identify players who are likely to benefit from a more structured approach to talent development. Based on exposure problems, the author intends to examine about the identification and development of Talent Sports football games based on the position of the 2018 PSGC Player. The research uses a pure description method. Data collection techniques using a sample of the entire sample because it is less than 100 people. The research participants were 22 PSGC players consisting of 2 people, 7 defensive midfielders for 10 people, or a midfielder and 3 attacking players. The results of this study illustrate how characteristics or characteristics (based on skills Anthropometry, physiological, psychological and basic techniques) are based on their position (goalkeeper, defender, midfielder or back or center, attacker or center forward). Physical condition level test eg Vo2 max, 30 meter running speed, agility, explosive limb muscles, speed dribbling skills, accuracy and speed of shooting, BMI (weight and height), long extremities, arm span, motivation level, and anxiety level. The results of this study illustrate how characteristics or characteristics (based on skills Anthropometry, physiological, psychological and basic techniques) are based on their position (goalkeeper, defender, midfielder or back or center, attacker or center forward). The trainer is expected to place players according to the identification and development of appropriate talent.
\end{abstract}

Keywords—talent identification; football sport; PSGC 2018

\section{INTRODUCTION}

Talent identification is a part that covers the anatomy and physiology and psychology of sports. Choosing the best person for sports, the main process in all countries is identification of talent. Identifying the best talent also enables countries to get limited resources for their athletes. Identification of scientific talent is an important factor in growing talented athletes in their fields so that they can achieve optimally. Measurement criteria for determining young players identified, using physical, physiological, and biomekannic examinations were provided.

"Anthropometric and physiological" [1], Psychological characteristics " [2], and" Technical Caracteristics "[3]. This review focuses on the anthropometric and physiological characteristics of soccer players with a view to establishing their role in identifying talent, talent search and development programs. Top-class soccer players must adapt to the physical demands of the game of football, which is multifactorial. Players may not need to have an extraordinary capacity in every field of physical performance but must have a fairly high level in all fields. This explains why there are clear individual differences in terms of anthropometry and physiological logical characteristics among top players. Various measurements have been used to evaluate specific aspects of the physical performance of teen and adult soccer players. The role of a player's position is related to him or his physiological capacity.

Studies in football are relatively small in number and variables in terms of psychological components are examined. That's the assumption that underlies the research on psychological characteristics in the scope of talent identification. This assumption has not been tested in football literature, but it is really important for all approaches to detecting it. Trainers and administrators interested in selecting elite players from the adult sub-elite may continue to test college-age players in hopes of identifying psychological factors that allow the best professional athletes to be chosen. Learning elite adult players to identify the psychological characteristics of young players does not seem to be a useful path for those interested in developing junior talent at 10, 12 or even 15 years of age.

An overview of identification talents in football uses physiological and technical testing procedures to summarize the issues associated with this process. Current research in soccer identification talent, among other sports, shows a systematic bias in selection towards players born early in the year and early maturity. From studies that investigate physiology (for example, strength) and technical (for example, dribbling), an individual has a tendency to perform better in this test so that it tends to become more influential in the game and is recognized as a more talented person.

When considering the current level of success in the future, elite youth play and the future of professional players score better in physiological and technical testing of youth recreation and future non-professional players, independently of maturity status. However, this testing procedure is not sensitive enough to distinguish young elite from sub-elite or future national teams from professional club players. Collectively, this study shows the need to use estimates of maturity status and subsequent appropriate data analysis obtained from physiological and technical testing. When maturity is taken into account, this testing procedure can provide an indication of responsiveness to training the load of young players and evaluating the potential to become a successful soccer player. 
However, this testing procedure should not be used as a marker of selection before full maturity in practice and must be part of a multidimensional approach to talent identification given the importance of other aspects of the game at the highest level (for example, cognitive intelligence, skills).

Identification of talent in football is followed by selection into a systematic program to develop the ability to play and maintain individuals to realize the potential that has been predicted. Identifying talent for field games at an early age is still far from analyzing the process. This is more complex in team games than in individual sports (eg track and field, swimming, rowing and cycling) where there are objective measures of performance. Furthermore, later success in football ultimately depends on a number of external factors, including the opportunity to practice, remain free from injury, the nature of guidance and coaching. Many of the proposed models are the best descriptive and schema and there has been no real effort to assess their validity. In this study, a multivariate approach to identity identification problems was adopted.

The team positions in football differ by denomination and defined general function. Few studies have investigated the physiological capacity profile representative of each. In most research studies, the players are classified into four groups: forwards/attackers, midfielders, defenders, and goalkeepers [4].

The author interpreted the position of the team in football as different from dominance and general functions defined. Several studies have investigated representative profiles of each physiological capacity. In most research studies, players are classified into four groups: front / attacker, midfielder, defender, and goalkeeper. However, it was not clear whether midfield players were selected as midfielders for their higher aerobic endurance capacity, or whether higher oxygen uptake was related to midfield playing positions or other factors. "In elite football, the players are the fastest players and time observations show that they run as long as a match." [5]. The authors interpret that in the future elite football is the fastest player and time observations show that they ran during the match.

Also, significant differences in age, stature, body mass and body mass index have been recently identified between elite players of different positions suggesting that players of particular size and shape may be suitable for the demands of the various playing positions [6].

So the explanation reinforces a study involving investigations from various players, positions, levels and competitions that have produced various motion time analysis reports. Also, significant differences in age, height, body mass and body mass index have been recently identified between elite players from different positions indicating that certain player sizes and shapes may not be suitable for the demands of various playing positions. In this case, the role of the position seems to have an influence on the total energy expenditure in the competition, showing different physical, physiological and bioenergy requirements by players from different positions. The overall biggest distance seems to be covered by midfield players who act as a connection between defense and attack.
An important task in sport science and performance analysis is to understand the relationship between skill acquisition and the development of talent and excellence. The development of theoretical principles to guide the design of skill acquisition programmes can also provide an informed basis for organising evaluation tests for talent identification and development in sport [7].

An important task in sports science and performance analysis is to understand the relationship between skill acquisition and talent development and excellence. The development of theoretical principles for guiding program design skills can also provide an information basis for organizing evaluation tests for identification of talent and development in sports. So both have important roles in developing skills.

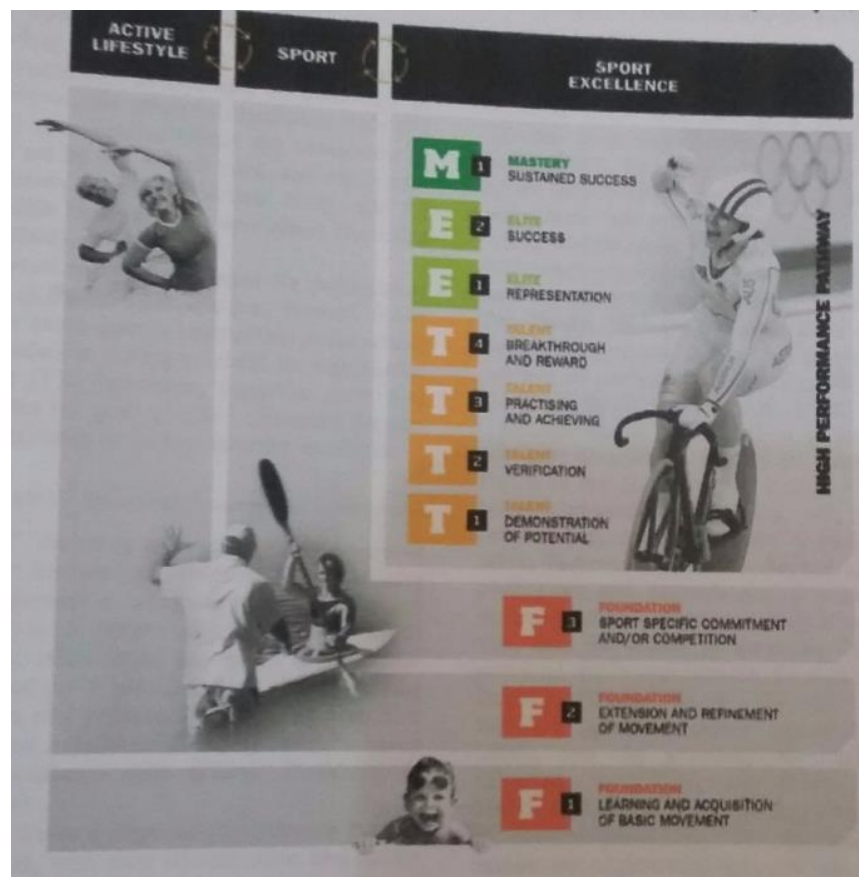

Fig. 1. FTEM [8].

These are Foundations (F1, F2 and F3); Talent (T1, T2, T3 and T4); Elite (E1 and E2); and Mastery (M) [8]. F1 is a Learning stage and the acquisition of a basic movement foundation (F1). Beginning to experience various movements that are capable of them various basic important movements. Extension and basic motion returns (F2), the characteristics of the F2 component are related to advancing and refining F1 movement experiences through extensive exposure to continuing formal and informal play, practice and games, both ways of specific sports and non-specific sports. Specific sports commitment and competition. The transition from F2 and becoming F3 is characterized by increased commitment to training, specific sports, skill development and official involvement in the competition. " $\mathrm{T}$ " is an element of proven talent and achievement.

High performance potential demonstration (T1). At the T1 level, athletes usually show gifts and talents that can be proven and measured in one or more of the physical, physiological, 
psychological, and skills domains, which show future potential in high per-centric sports. This can occur through formal or informal talent in the identification process. Talent Verification (T2), The technical or talent verification (T2) are seen as sequential and complementary for T1, where evidence-based observations (T1) must be supplemented by judgment and intuition from experienced experienced trainers. Practicing and Achievement (T3), Having gained increased talent, coaches or national sports organizations, athletes are now comitted to higher levels of certain sporting practices and striving for continuous improvement in performance that focuses on benchmark results (See T4). Breakthroughs and prizes (T4), getting professional support to continue developing is a key feature of T4. The progress of athletes in improving their appearance during $\mathrm{T} 3$ is basically rewarded at T4 where they can get a sports scholarship at a University or Sports Academy or institution, or be recruited into a professional team or training squad elite, greatly enhancing their development progress.

Elite senior representation (E1), senior elite success (E2), and sustained elite success or mastery (M) Distinguishing between good and large elite athletes in the field of talent development is important to understanding the characteristics of their expertise and development [8]. However, as shown in Table I, there can be a lack of consistency in defining "elite" performance, with a lack of differentiation and breadth of being ordinary. In accordance with the views of practitioners of development, elite performance in the FTEM framework is represented by performance benchmarks that clearly develop mentally and achieved planning results. To maximize utility from the FTEM framework, the final characterization of elite players depends on whether athletes are predominantly involved in Olympic sports or professional sports. For Olympic sports, athletes at E1 are defined as representing their country at the senior international level; people at E2 are defined as achieving podium success in large senior international competitions (for example, Olympics or World Championships); and they reach $\mathrm{M}$ or mastery level, succeed at E2 level over several high performance cycles (i.e., a typical 8year period based on two four-year high performance cycles). Mastery as the end.

An illustration of the framework is represented in figure 1.2. Talent development is "Three stages of talent development are recognized (Initiation, Development \& Perfection). "Transition between stages is not determined by time but is contingent on tasks achieved and relationships or measures developed." [8]. Three stages of development of recognized talents (initiation, development \& perfection). The transition between stages is not determined by chronological age but depends on learning and the tasks achieved \& relationships or attitudes developed. Expertise is the final product of an extensive number of deliberate practices of 10,000 goaloriented hours, effortful practices that are not inherently fun, or intrinsically motivating. A monotonous relationship is concluded where the difference in investment practices equates different levels of performance.

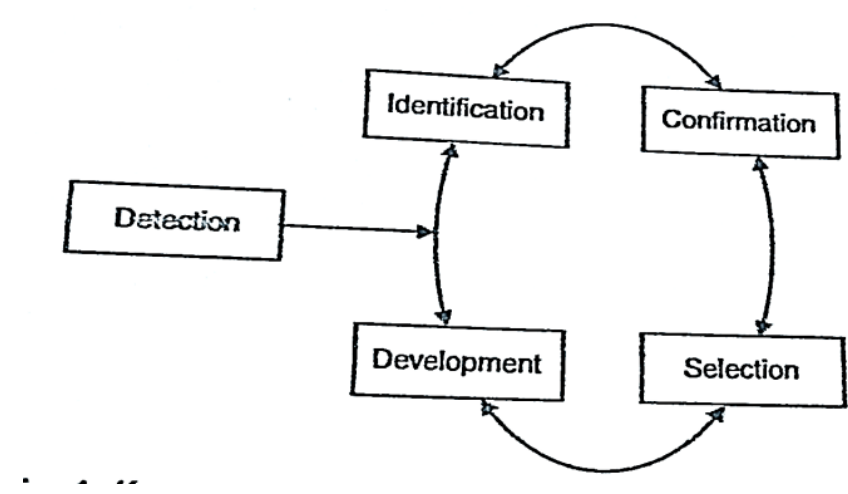

Fig. 2. Relationship of talent identification and development [9].

The relationship between talent identification and talent development is the key stage in the process of identifying and developing talent. Detection is the discovery of potential players who are not involved in sports; choices involve choosing the most appropriate athlete (group) to complete a particular task (in a team). However, even though talent identification and development programs have gained popularity in recent decades, there remains a lack of consensus in relation to how talent must be determined or identified and there is no uniformly accepted theoretical framework to guide current practice. The success rate of talent identification and program development is rarely assessed and the validity of the model is applied to remnants that are highly debated. This article provides an overview of current knowledge in this area with a special focus on issues related to the identification of gifted adolescents. There is growing agreement that traditional cross-sectional talent identification models tend to exclude many, especially late maturity, 'promising' children from development programs because of the dynamic and multidimensional nature of sports talent. A conceptual framework that recognizes genetic and environmental influences and considers the dynamic and multidimensional nature of sports talent presented. The relevance of this model is highlighted and recommendations for future work are provided. This suggests that identification of talent and development programs must be dynamic and interconnected taking into account the status of maturity and the potential to develop rather than to exclude children at an early age. Finally, more representative tasks must be developed and used in multidimensional designs to improve the effectiveness of talent identification and program development.

Finally a time-motion analysis study in soccer has reported a summary of physical requirements by reporting overall frequency, total and mean duration of movement, physiological average and peak output and the number of closed distances. For the advancement of knowledge it is important to further investigate aspects of the match and provide a higher level of detail. "The average work intensity during a typical football game between the elite male level senior teams is approximately $85 \%$ of the maximal heart rate (HRmax), which corresponds to about $75 \%$ of maximum oxygen uptake (VO2max)" [10]. Football played at relatively high average work intensity interspersed with very short periods of time high intensity of running and jumping can be considered as sports with both aerobic and anaerobic demands. The average 
work intensity during a typical soccer match between elite male teams at the senior level is around $85 \%$ of the maximum heart rate (HRmax), which is associated with about $75 \%$ of maximal oxygen uptake (VO2max). Anthropometry "Forms of body size that influence achievement, such as: Body Height (TB), Body Weight (BB), Body Mass Index (BMI), Arm Length (PRL), and Leg Length (PT).

Being a star player is not easy, because the player must master good technique, tactics, mental and physical. These factors must be developed if you want to achieve maximum achievement. When soccer matches take place, the players need good physical quality because most of the movements carried out in soccer are basic movements, whether they are motion or technique without balls or movement or technique with the ball. A player must be able to run both with short and long steps, because he has to change the running speed. Other movements such as walking, tiptoeing, jumping, jumping, rolling, spinning, and stopping suddenly all of which must be mastered by soccer players. that the basic movement consists of: "running movements, stopping movements, and running suddenly (stopping and starting), turning movements, and rotating movements, jumping / jumping movements" [11]. Basic ability is very closely related to the ability to coordinate physical, tactical and mental movements. In order to have good basic abilities players must also have this coordination ability. Basic skills must be truly mastered and learned early to develop a game of soccer players. This is one of the factors that determine a team's win or lose in a match. Many factors must be considered to improve football performance, including infrastructure, quality coaches, talented players, and regular competition and must be supported by adequate science, knowledge and technology.

Galuh Ciamis Football Association (abbreviated as PSGC Ciamis) is an Indonesian football team from West Java based at Galuh Stadium, Ciamis Regency, West Java, Indonesia. PSGC currently competes in the Indonesian League First Division. In 2016 the PSGC Ciamis joined the Indonesia Soccer Champioship B Division First Division. The club has entered the professional league in the Indonesian football arena. Evidenced in League 2 Indonesia went through to the big 16. Scouting programs and the development of talented athletes in advanced countries have been carried out by gaining adequate resources, including not only government and community funds, but also expert support through a cross-disciplinary and interdisciplinary approach. Sophistication in the field of measurement and evaluation and the discovery of instruments that can be used to predict one's achievements encourage us to work effectively in identifying and selecting talented athletes.

It is realized that efforts to achieve achievements in sports are complex, because they involve many factors including internal factors such as: physical and mental athletes and external factors such as: the natural environment and equipment. Internal factors are actually derived from the quality of the athlete itself, where qualified athletes have the potential to be innate (talent) in accordance with the demands of the sport and ready to be developed to achieve peak performance. Experience shows that only athletes who are talented and want to practice well can achieve peak performance (peack performance). Peak achievement is the result of all the coaching programs in a certain period of time which are a mixture of training processes that are systematically designed, tiered, interrelated, repetitive and increasingly increasing.

Players who are quite experienced in the Indonesian League have their respective talents. The majority of players came from Ciamis Regency who had defended PORDA 2014, PORDA 2018 and as Runner up West Java 2014. Players who came from SSB Ciamis started their football with the majority of elementary school age. Measurements for each participant were carried out according to four categories. Anthropometry players who have a variety of anthropometric characters are high, medium, etc., physiological, and psychological profiles and performance of soccer-specific skills. The PSGC team is a benchmark for SSB in Ciamis Regency because it has a professional level and the players are the best choice in Ciamis Regency. In 2018, 100 percent of PSGC players are local native players.

In many electoral districts players are based on natural talent without having analyzed the player's position needs. In fact, there are trainers who position their players according to the wishes of the child or player, even if they are positioned in the right place that should be more feasible, maybe the player will develop better according to his talent. So that when they are in the Soeratin Cup selection, PORDA even PSGC even though there is no need to select from the beginning because they have been provided before.

A key problem for these clubs is identifying those players most likely to benefit from this more structured approach to talent development. Thus far in most clubs, players are selected on the initial recommendation of talent scouts with progress and eventual contractual obligation being dependent on performance in training and trial matches" [12].

The main problem for these clubs is identifying the players who are most likely to benefit from this more structured approach to talent development. As far as in most clubs, players selected on the initial recommendations of talent seekers with progress and contractual obligations ultimately depend on performance in training and trial matches. Especially in preparation at SSB, children are equipped with talent that is in accordance with their position so that when they stay in the club they can continue training. and Based on the explanation of the problem the author intends to examine the Identification and Development of Talent Sports Branch Football Games Based on the Position of PSGC 2018 Players.

\section{METHOD}
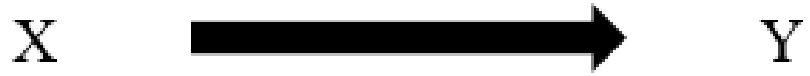

Fig. 3. Descriptive Research Design (Sugiyono, 2014, p.42).

The word population (population) is a term used in research that refers to a group of individuals with distinctive characteristics that are of concern in a study. Fraenkel and Wallen, defines that the population is all members of a particular group that concern the researcher and become the 
subject of the generalization of the results of the study [13]. From the definition above, the population used in this study were 22 PSGC players.

"Samples are partially taken from the entire object under study and are considered to represent the entire population" [14]. "The sampling technique in this study is total sampling. Total sampling is a sampling technique where the number of samples is the same as the population " [15]. The reason for taking total sampling is because according to Sugiyono the total population of less than 100 all of the population is all research samples [15].

Team positions in football are different from dominance and general functions are defined. Several studies have investigated representative profiles of each physiological capacity. In most research studies, players are classified into four groups: forward / attacker, midfielder, defender, and goalkeeper [4]. "Consequently, multiple factors together with the influence of growth and maturation across the developmental cycle" [16]. As a result, any attempt to identify early soccer talent must take into account these several factors along with the influence of growth and maturation throughout the development cycle. Equipped with age training or trainning age.

\section{RESULT AND DISCUSSION}

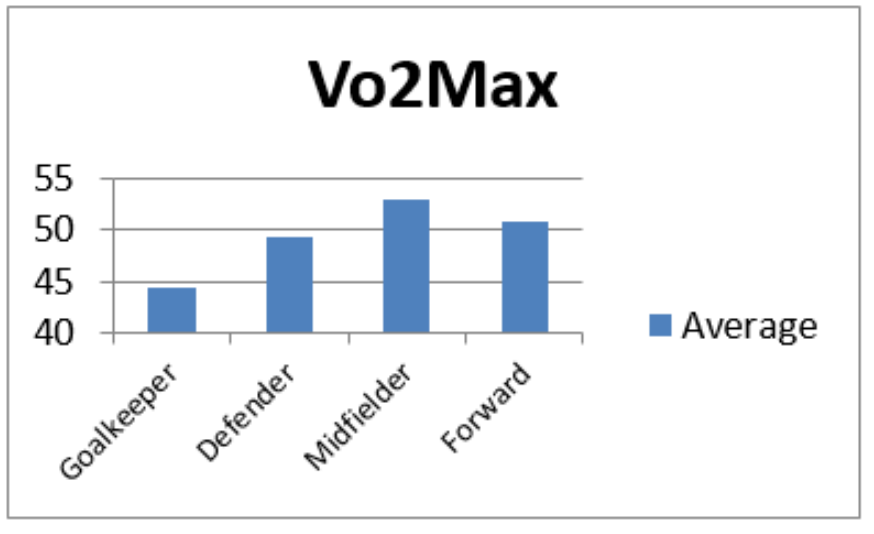

Source, Results of Author Data Processing

Fig. 4. Figure of vo2max level diagram based on player position.

Based on this explanation, the authors concluded that overall the goalkeeper has VO2max of $44.35<45$, which means the VO2max level of the goalkeeper is below the average criteria. The defender has VO2max of 49> 53 which means the VO2max level of the defender is in the above average criteria. The midfielder has VO2max of 53>53 meaning the VO2max level of the midfielder is included in the special criteria. The forward has VO2max of $51<45$ which means that the forward level of $\mathrm{VO} 2 \mathrm{max}$ is in the above average criteria.

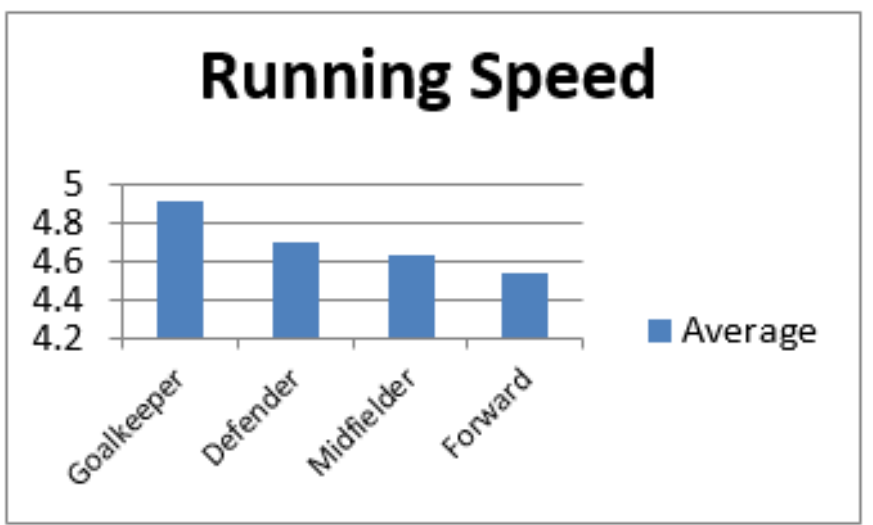

Source, Results of Author Data Processing

Fig. 5. Figure of Running Speed Level Chart Based on Player Position in seconds.

Based on this explanation the author concludes that overall the goalkeeper has a 30 meter running speed of $4.91(5.15$ $<x \geq 4.89$ ) which means the 30 meter running speed of the goalkeeper is in the low criteria. The defender has a 30 meter running speed of $4.81(4.89<x \geq 4.64)$, which means that the level of running speed is 30 meters. The midfielder has a 30 meter running speed of $4.64(4.89<\mathrm{x} \geq 4.64)$ which means the 30 meter running speed of the midfielder is in the medium criteria. The forward has a level of 30 meters running speed of 4.54 (4.64 $<\mathrm{x} \geq 4.38$ ), which means the level of the 30 -meter running speed of the forward is in high criteria.

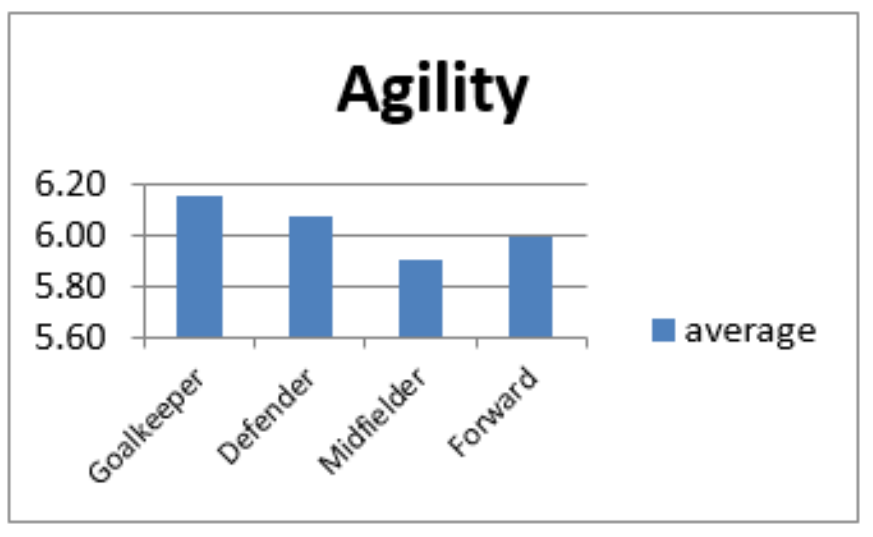

Source, Results of Author Data Processin

Fig. 6. Picture of agility level diagram based on player position in seconds.

Based on this explanation the author concludes that overall the goalkeeper has an agility level of $6.156 .25<\mathrm{x} \geq 5.71$ ) means the level of agility of the goalkeeper is in the criteria of being. Defender has agility of $4.81(4.89<\mathrm{x} \geq 4.64)$ means that the level of agility of the defender is in the criteria of being. The midfielder has agility of $4.64(4.89<\mathrm{x} \geq 4.64)$, meaning the level of agility of the midfielder is in the criteria of being. The forward has an agility level of 4.54 (4.64 $<\mathrm{x} \geq 4.38)$, which means that the level of agility of the forwards is high. 


\section{Leg Muscle Explosion}

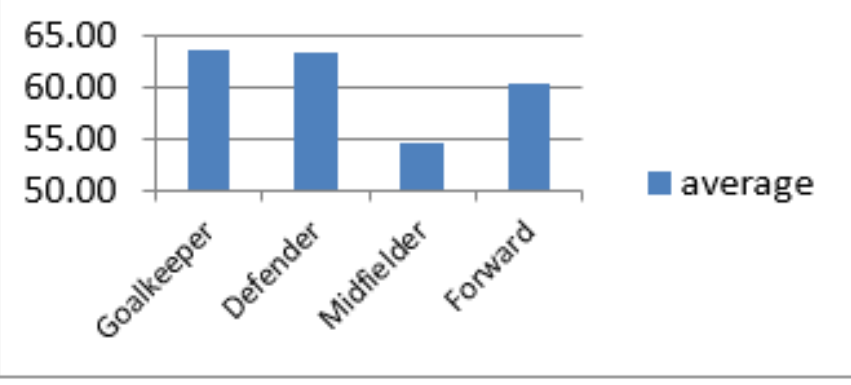

Source, Results of Author Data Processing

Fig. 7. Picture of leg muscle explosion power level chart based on player position.

Based on this explanation, the authors concluded that overall the goalkeeper has a leg muscle explosive power of $63.5(62.15<x \leq 68.45)$, which means that the goalkeeper's muscle explosive power level is in the high criteria. Defender has a leg muscle explosive power of $63.43(62.15<\mathrm{x} \leq 68.45)$ which means that the level of explosive muscle leg power of the defender is in high criteria. The midfielder has a limb muscle explosive power of $54.60(49.55<x \leq 55.85)$, which means that the level of explosive muscle leg power in the middle players is in the low criteria. The forward has a leg muscle explosive power level of 60.33 (55.85 $<x \leq 62.15)$, which means that the level of explosive limb muscle explosive power is moderate.

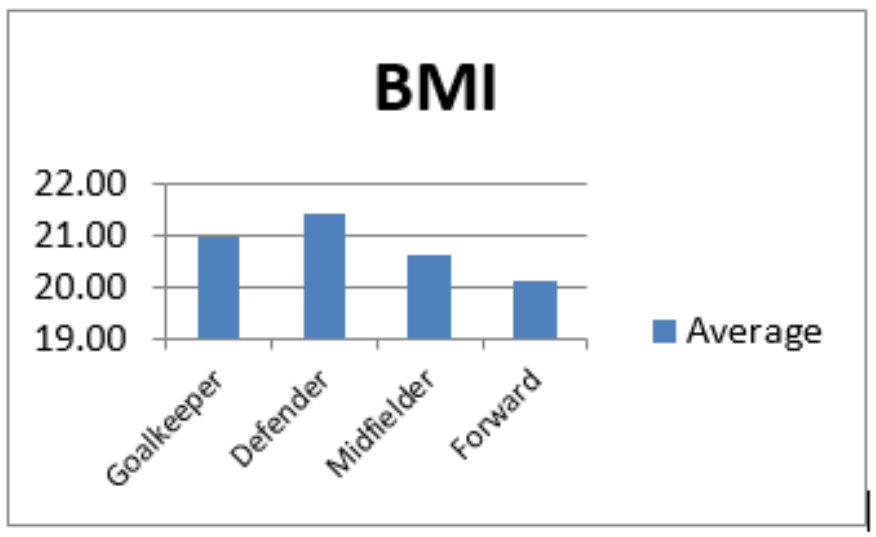

Source, Results of Author Data Processing

Fig. 8. Bmi level chart based on player position.

Based on this explanation, the authors concluded that overall the goalkeeper has a BMI of 20.95 (18.5-24.9), which means the BMI of the goalkeeper is in very low criteria. Defender has a BMI of 21.44 (18.5-24.9) means that the BMI level of a defender's ball is in the criteria of being. The midfielder has a BMI of 20.64 (18.5-24.9), meaning the midfielder's BMI level is in the medium criteria. The forward has a BMI of $20.13(18.5-24.9)$ which means that the forward $\mathrm{BMI}$ level is high.

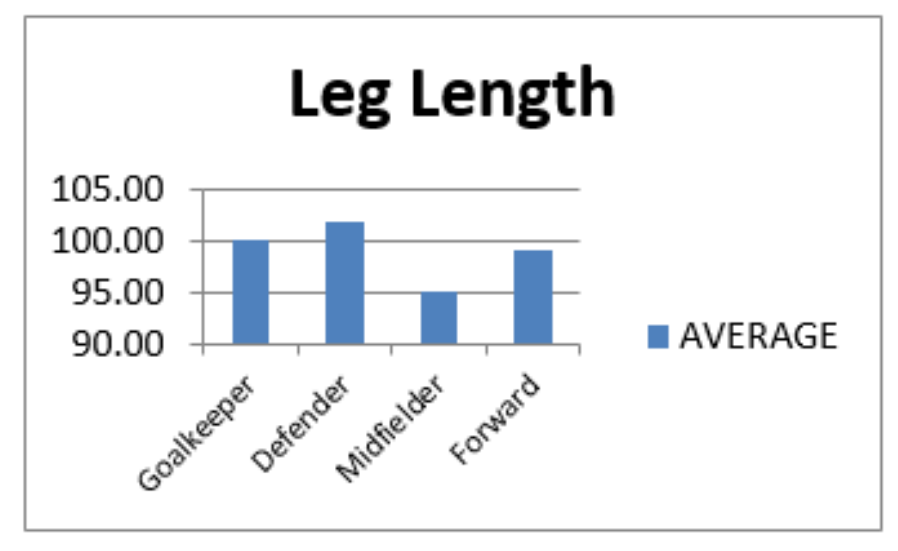

Source, Results of Author Data Processing

Fig. 9. Leg length level diagram based on player position.

Based on this explanation, the authors concluded that overall the goalkeeper has a leg length of $100(100<x \leq 105)$, which means that the goalkeeper's long leg skill level is included in the length criteria. The defender has a leg length of $101.7(100<\mathrm{x} \leq 105)$, meaning that the length of the leg of the defender is in the long criteria. The midfielder has a leg length of $95.1(95<x \leq 100)$, which means the length of the leg of the midfielder is in the criteria of being. The forward has a leg length level of $99(95<x \leq 100)$, which means that the level of the length of the forward limbs is in the criteria of being.

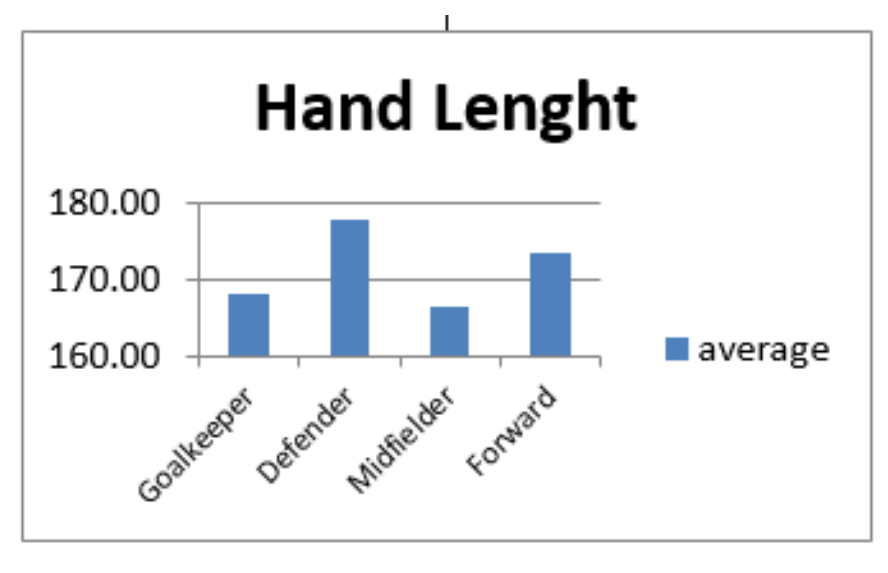

Source, Results of Author Data Processing

Fig. 10. Hand length level diagram based on player position.

Based on this explanation the author concludes that overall the goalkeeper has a length of arm range of $168(166<x \leq 175)$ which means the skill level of the length of the goalkeeper's arm is in the criteria of being. Defender has a long arm span of $177(175<x \leq 185)$, meaning that the level of the length of the range of the defender's arm is in the long criteria. The midfielder has a long arm span of $166.6(175<\mathrm{x} \leq 185)$, which means that the level of the length of the arm span of the midfielder is in the criteria of being. The forward has a level of arm length range of $99(175<x \leq 185)$, which means the level of the length of the range of the forward arm is in the medium criteria. 


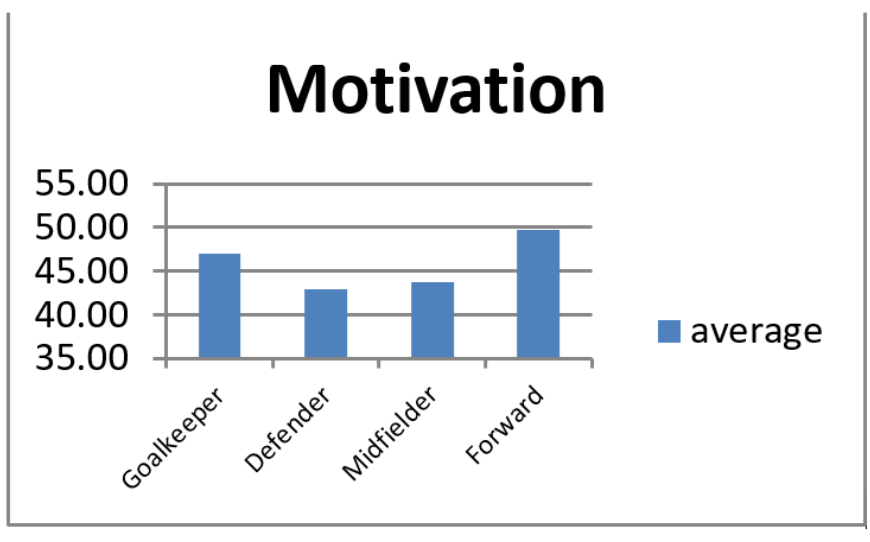

Source, Results of Author Data Processing

Fig. 11. Motivation level chart based on player position.

Based on this explanation the author concludes that overall the goalkeeper has a motivation level of $47(46.43<\mathrm{x} \leq 50.12)$, meaning the level of skill of the goalkeeper's motivation level is in the high criteria. Defender has a motivation level of 43 $(43.75<x \leq 46.43)$ which means that the motivation level of the defender is in the medium criterion. The midfielder has a motivation level of $43.7(43.75<\mathrm{x} \leq 46.43)$, which means that the motivation level of the midfielder is in the medium criteria. The forward has a motivation level of $49.67(46.43<\mathrm{x} \leq 50.12)$ which means that the motivation level of the forwards is high.

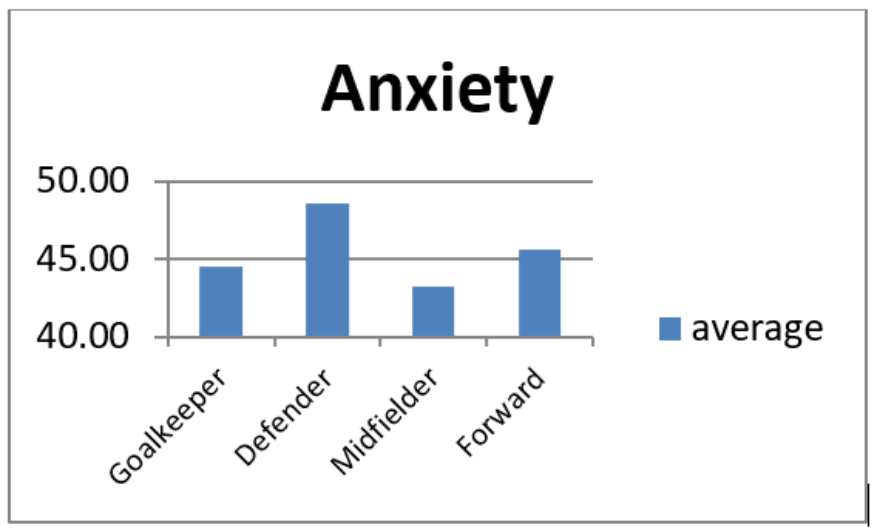

Source, Results of Author Data Processing

Fig. 12. Picture of anxiety level diagram based on player position.

Based on this explanation the author concludes that overall the goalkeeper has an anxiety level of $44.5(47.27<\mathrm{x} \leq 51.08)$ meaning the level of anxiety of the goalkeeper is in the high criteria. Defenders have an anxiety level of 48.57 (47.27 $<\mathrm{x} \leq 51.08$ ) meaning that the level of anxiety of the defender is in high criteria. The midfielder has an anxiety level of 43.7 $(43.46<x \leq 42.27)$ means the level of anxiety of the midfielder is in the criteria of being. The forward has an anxiety level of $45.67(47.27<\mathrm{x} \leq 51.08)$, which means that the level of anxiety of the forwards is high.

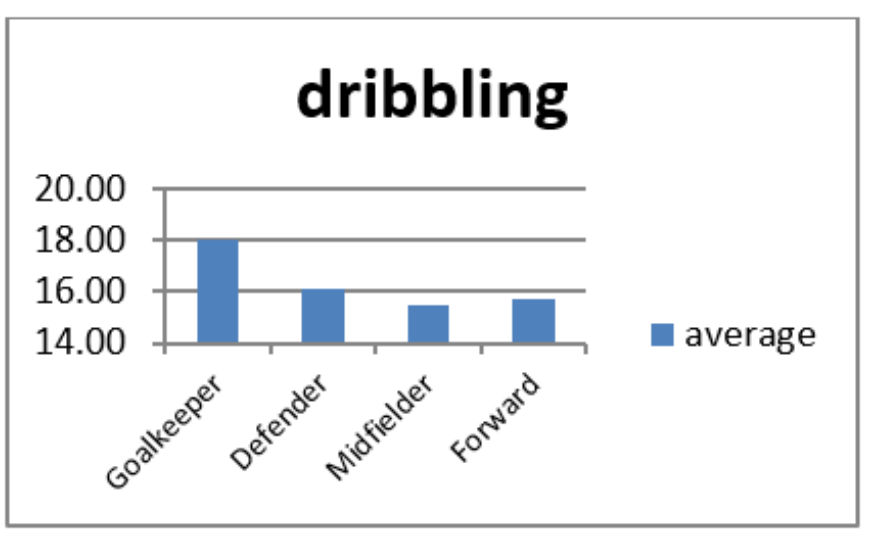

Source, Results of Author Data Processing

Fig. 13. Picture of ball dribbling level diagram based on player position.

Based on these explanations the author concluded that overall the goalkeeper has a dribbling skill of $18(<17.58)$, which means the goalkeeper's skill level is in very low criteria. Defender has a dribbling skill of $16.14 \quad(16.5<\mathrm{x} \geq 15,42)$ meaning that the defender's level of dribbling skill is in the medium criteria. The midfielder has dribbling skills of 15.5 (16.5 $<x \geq 15.42$ ), meaning the level of dribbling skill in the midfielder is in the medium criteria. The forward has a dribbling skill level of $15.68(16.5<x \geq 15.42)$, which means the skill level is dribbling forward in midfield.

\section{Shooting Accuracy}

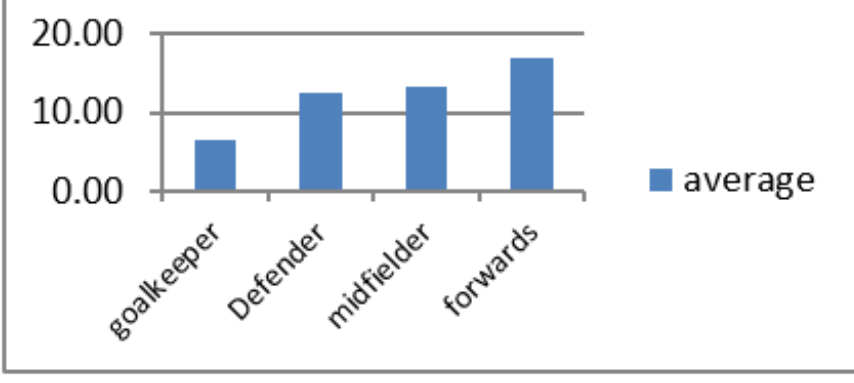

Source, Results of Author Data Processing

Fig. 14. Shooting accuracy level chart based on player position.

Based on this explanation, the author concluded that overall the goalkeeper has a shooting accuracy of 6.5 (<7.62), meaning that the goalkeeper's shooting accuracy is in a very low criterion. The defender has the skill of shooting accuracy of $12.57(11.21<\mathrm{x} \leq 14.79)$, meaning that the level of accuracy of the shooting of the defender's ball is in the criteria of being. The midfielder has the skill of shooting accuracy of 13.4 (11.21 $<\mathrm{x} \leq 14.79$ ), which means that the skill level of accuracy of the midfielder's shooting is in the criteria of being. The forward has a skill level of shooting accuracy of 17 (14.79 $<\mathrm{x} \leq 18.38$ ), which means the skill level of the accuracy of the forward shooting is high. 


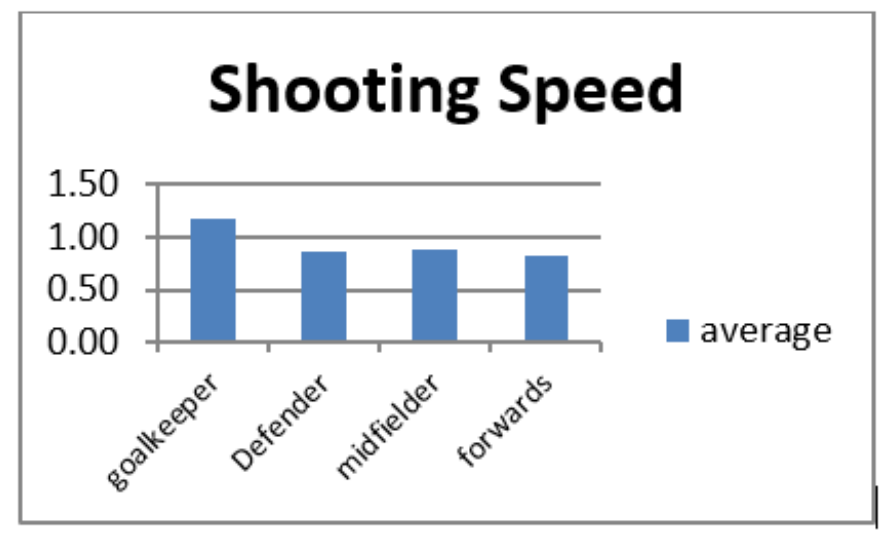

Source, Results of Author Data Processing

Fig. 15. Picture of shooting speed level diagram based on player position

Based on this explanation the author concluded that overall the goalkeeper has a shooting speed of $1.18(<1.01)$, meaning that the skill level of the goalkeeper shooting speed is in very low criteria. Defender has the skill of shooting speed of 0.87 $(0.83<\mathrm{x} \leq 0.95)$ means that the speed of shooting of the defender's ball is in the criteria of being. The midfielder has the skill of shooting speed of $0.88(0.83<\mathrm{x} \leq 0.95)$, meaning that the level of skill of the shooting speed of the midfielder is in the criteria of being. The forward has a skill level of shooting speed of $0.81(0.70<\mathrm{x}<0.83)$ which means that the skill level of the forward player's shooting speed is high.

\section{CONCLUSION}

Based on calculations and data analysis, the authors concluded that the goalkeeper had physiological levels with VO2max criteria with a criterion of $<45$ which means that the VO2max goalkeeper was below average. The goalkeeper has a low running speed criterion. The agility of a goalkeeper is included in the medium category. For leg muscle explosive power a goalkeeper is included in the high category. In speed the dribbling skills are categorized as low. The accuracy and speed of shooting also have a low level. The BMI of a goalkeeper must be in the ideal weight category with a relatively long leg length and a medium arm span. The level of motivation and anxiety are equally high.

Defenders have physiological levels with VO2max criteria with a criterion of $<53$ which means that VO2max of the defender is above average. Defender has the criteria for moderate running speed. The agility of a defender is included in the medium category. For leg muscle explosive power, a defender is in the high category. In speed the dribbling skills are categorized as being moderate. The accuracy and speed of shooting also have a moderate level. The BMI of a defender must be in the ideal weight category with a relatively long leg length and a medium arm span. Moderate motivation level and high anxiety level.

Midfielders have physiological levels with VO2max criteria with criteria> 53, which means that VO2max is a special midfielder. Midfielders have run speed criteria with moderate criteria. The agility of a midfielder is in the moderate category. For the explosive power of the limbs of a midfielder, it is a low category. In speed the dribbling skills are categorized as being moderate. The accuracy and speed of shooting also have a moderate level. A midfielder's BMI must be in the ideal weight category, with a relatively long leg length and a medium arm span. Moderate level of motivation and level of anxiety.

Forwards have physiological levels with VO2max criteria with criteria $>49$, which means that VO2max forwards is above average. The forward has high criteria for running speed. The agility of a forward is a high category. For the explosive power of the limbs of a forward player, it is in the medium category. In speed the dribbling skills are categorized as being moderate. The accuracy and speed of shooting also have a high level. A forward player's BMI must be in the ideal weight category, with a relatively long leg length and a medium arm span. High levels of motivation and anxiety levels.

\section{REFERENCES}

[1] T. Reilly, J. Bangsbo and Franks, A. (2013). Anthropometric and physiological predispositions for elite soccer Anthropometric and physiological predispositions for elite soccer, (March 2013), 37-41.

[2] Morris, T. (2010). Psychological characteristics and talent identification in soccer Psychological characteristics and talent identi ${ }^{\circledR}$ cation in soccer, (August 2013), 37-41. https://doi.org/10.1080/02640410050120096.

[3] Meylan, C., Cronin, J., Oliver, J., \& Hughes, M. (2010). Talen Identification in Soccer: The Role of Maturity Status on Physical , Physiological and Technical Characteristics, 5(4), 571-592.

[4] Bush, M., Barnes, C., Archer, D. T., Hogg, B., \& Bradley, P. S. (2015). Human Movement Science Evolution of match performance parameters for various playing positions in the English Premier League. HUMAN MOVEMENT SCIENCE, $39, \quad 1-11$. https://doi.org/10.1016/j.humov.2014.10.003.

[5] Rienzi E, Drust B, Reilly T, Carter JE, M. A. (2000). Investigation of anthropometric and work-rate profiles of elite South American international soccer players. J Sports Med Phys Fitness, 2.

[6] Bloomfield, J. P., Barker, J. A., \& Robinson, N. (2005). Modeling Fracture Porosity Development Using Simple Growth Laws, 43(3), 314 326.

[7] Davids, K., Araújo, D., Vilar, L., Renshaw, I., \& Pinder, R. (2013). An Ecological Dynamics Approach to Skill Acquisition: Implications for Development of Talent in Sport. Talent Development \& Excellence, 5(1), 21-34

[8] Gulbin, J. P., Croser, M. J., Morley, E. J., \& Weissensteiner, J. (2013). An integrated framework for the optimisation of sport and athlete development: A practitioner approach An integrated framework for the optimisation of sport and athlete, (August), 37-41.[4] https://doi.org/10.1080/02640414.2013.781661.

[9] Vaeyens, R., Lenoir, M., Williams, A. M., \& Philippaerts, R. M. (2008). Talent Identification and Development Programmes in Sport Current Models and Future Directions, 38(9), 703-714.

[10] Stolen, T., Chamari, K., Castagna, C., \& Wisloff, U. (2005). Physiology of Soccer. Sport Medicine, 35(6), 501-536.

[11] Suwarno. (2001). Sepakbola (Gerakan Dasar dan Teknik Dasar). Yogyakarta.

[12] Williams, A. M. (2010). Perceptual skill in soccer: Implications for talent identification and development Perceptual skill in soccer: Implications for talent identification and development. Journal of Sports Sciences, (March 2013), 37-41.

[13] Fraenkel, J., \& Wallen, N. (2008). How to Design and Evaluate Research in Education. New York: McGraw-Hill Higher Education.[14]

[14] S. Notoatmodjo, Promosi kesehatan teori dan aplikasi. Jakarta: Rineka Cipta, 52-54, 2005. 
[15] Sugiyono, Metode Penelitian Kuantitatif Kualitatif dan R\&D. Bandung: Alfabeta, 2014

[16] Hoare, D. G., \& Warr, C. R. (2000). Talent identification and women 's soccer : An Australian experience Talent identi ${ }^{\circledR}$ cation and women's soccer: An Australian experience. Journal of Sports Sciences, 18(October 2012), 37-41. 